\title{
Creatividad, creencias paranormales y deterioro cognitivo en personas mayores
}

\author{
Inmaculada Méndez ${ }^{1}$, Julia García-Sevilla ${ }^{1}$, Juan P. Martínez ${ }^{1}$, Isabel \\ García-Munuera ${ }^{2}$, Ana $\mathrm{M}^{\mathrm{a}}$ Bermúdez $^{1}$ y Pilar Pérez ${ }^{1}$ \\ ${ }^{1}$ Universidad de Murcia (España); ${ }^{2}$ Universidad de Granada (España)
}

En el caso de las personas mayores, el pensamiento estético y la creatividad son formas de mantener activas la mente y la imaginación, contribuyendo a prevenir el deterioro cognitivo. Asimismo tener un sistema de creencias religiosas o incluso un sistema de creencias o experiencias paranormales ha demostrado ser beneficioso sobre la salud de los ancianos. El objetivo del estudio fue estudiar la relación entre la creatividad, el grado de deterioro cognitivo y las creencias paranormales. Se analizaron si existían diferencias atendiendo a características sociodemográficas. A un grupo de 36 personas mayores (16 varones) con edades comprendidas entre los 66 y 95 años se le administró el Mini-Examen-Cognoscitivo (MEC), el Test CREA y la Escala Mejorada de Creencias Paranormales. Los resultados principales destacan que: los sujetos con mejor estado cognitivo son los más creativos; los menos creativos poseían más Creencias religiosas tradicionales e incluso mayor superstición; los ancianos con mejor estado cognitivo eran los menos supersticiosos. Es destacable que los varones eran más creativos, mientras que las mujeres destacaban en creencias religiosas tradicionales. El estudio permite reflexionar sobre la importancia de que los profesionales que trabajen con personas mayores lleven a cabo programas que favorezcan la creatividad e incluso recaben información sobre el sistema de creencias.

Palabras clave: Parapsicología, superstición, creatividad, deterioro cognitivo, persona mayor.

Creativity, paranormal beliefs and cognitive impairment in the elderly. In the case of older people, aesthetic thought and creativity are ways to keep mind and imagination active by contributing to prevent cognitive decline. Furthermore, having a religious belief system or even a belief system or paranormal experiences has proved to be beneficial for the health of the elderly. The objective was to study the relationship between creativity, degree of cognitive impairment and paranormal beliefs. The existence of differences depending on sociodemographic characteristics was analyzed. A group of 36 elderly people (16 men) aged between 66 and 95 years were administered the Mini-Mental State Examination (MEC), the CREA Test and the Paranormal Beliefs Scale. The main outcomes include that: those with better cognitive status are more creative; the less creative subjects had more traditional religious beliefs and even greater superstition; the elderly with better cognitive status were the least superstitious. It is remarkable that men were more creative, while women excelled in traditional religious beliefs. The study allows reflecting on the importance of professionals who work with the elderly to carry out programs to encourage creativity and even collect information about the belief system.

Keywords: Parapsychology, superstition, creativity, cognitive impairment, elderly.

Correspondencia: Inmaculada Méndez. Facultad de Psicología. Departamento de Psicología Evolutiva y de la Educación. Universidad de Murcia. Campus de Espinardo. C.P. 30100. Murcia (España).E-mail. inmamendez@um.es 
La consecuencia más inmediata del progresivo envejecimiento de la población actual es el incremento de la dependencia (Gázquez, Pérez-Fuentes, Mercader, y Molero, 2011) ante lo cual, los estudios realizados en nuestro país ponen de manifiesto que los centros institucionalizados son los recursos entre los que existen más beneficiarios (Pérez-Fuentes, Molero, y Gázquez, 2011). Lo que unido al aumento de la esperanza de vida ha dado lugar a acciones e investigaciones encaminadas a la mejora de la atención a los mayores y su calidad de vida (Carrascal y Solera, 2014). Los estudios en gerontología están promoviendo un envejecimiento activo, lo que supone mantener al anciano activo a nivel mental, físico y social (Fernández-Ballesteros, Caprara, Íñiguez, y García, 2005). Fernández-Ballesteros et al. (2005) destacan que durante la vejez el aprendizaje es posible, dada la plasticidad neuronal, siendo beneficioso puesto que frena el declive a nivel cognitivo. Por este motivo, diversos autores (Carrascal y Solera, 2014; García-Sevilla, Fernández, Fuentes, López, y Moreno, 2014) destacan la importancia de las diferentes estrategias de estimulación cognitiva o de psicoestimulación para intentar mantener las funciones cognitivas en la vejez evitando el deterioro, fomentado la independencia y la calidad de vida. Dichos programas de entrenamiento cognitivo han estado centrados sobre todo en la memoria, lectura, atención, escritura, etc. a través de diferentes formatos tales como: lápiz y papel, soporte informático, tableros de orientación e incluso a través del panel de psicoestimulación (Abellán, García-Sevilla, y Méndez, 2013) o recientemente implantando programas conjuntos de estimulación cognitiva e inteligencia emocional (Pérez-Fuentes, Molero, Gázquez, y Soler, 2014). Carrascal y Solera (2014) abogan que los programas de estimulación artística como la arteterapia, la danzaterapia u otras técnicas de desarrollo y motivación creativas, han mostrado fomentar un estado activo de la mente del individuo al favorecer la fluidez de ideas, sentimientos y las emociones. Es por ello que sugieren que en los casos de deterioro físico o cognitivo, dichas estrategias permiten llevar a cabo un proceso de estimulación pasiva, a través de la exposición de diferentes estímulos captando la atención sensorial. Asimismo, la realización de actividades constructivas, que supongan la creación o producción propia derivada del intercambio artístico con el medio social, fomentaría sus capacidades cognitivas, afectivas y sociales, mejorando por tanto su calidad de vida.

A este respecto, Carrascal y Solera (2014) destacan que la creatividad, entendida como capacidad de generar ideas o resolver problemas, está implicada en el desarrollo de los sujetos, siendo una parte fundamental del potencial humano, que si es promovida a lo largo de la vida le permitiría transformar, cambiar y mejorar su existencia. En los ancianos, según Gardner (2010) no es tan significativo el hecho de crear nuevos productos, plantear nuevas cuestiones o definir nuevos problemas, sino que cobran importancia la exploración y el descubrimiento. El pensamiento estético y la 
creatividad permiten mantener activa la mente y la imaginación, previniendo el deterioro cognitivo en los ancianos (Carrascal y Solera, 2014).

López-Pousa (2012) resaltan que diversos estudios han puesto de manifiesto que, dependiendo de la enfermedad neurodegenerativa la afectación de la creatividad varía. Existen casos clínicos en los cuales durante la enfermedad se habrían producido cambios en la capacidad y producción artística, por ejemplo, en los ancianos con Enfermedad de Alzheimer (EA) se produce un abandono de la producción artística a favor de la copia, sin embargo en las demencias con cuerpos de Lewy (DCL) se ha demostrado una mayor capacidad creativa.

Por otro lado, hay ancianos que para adaptarse al proceso de envejecimiento, la enfermedad y el sufrimiento buscan apoyo en las creencias religiosas, ya que les dota de esperanza y autoestima, promoviendo un soporte socioemocional y favoreciendo los mecanismos psicológicos adecuados (Reyes-Ortiz, 1998). Unido a lo anterior, según Parra y Corbetta (2013) aquellos sujetos interesados en temas paranormales y que han vivido una o varias experiencias que interpretan como tales, determinaron que dichas situaciones habían reforzado sus creencias espirituales así como su percepción subjetiva de bienestar. Del mismo modo, tener un sistema de creencias o experiencias paranormales, esto es, interpretaciones más plancenteras de la realidad, ha mostrado ser beneficioso sobre la salud de los ancianos; por ejemplo, disminuyen el riesgo de mortalidad por problemas cardiacos, disminuyen la ansiedad y la depresión; aumentan el nivel de satisfacción de vida así como el bienestar, etc. Por lo que autores como Krippner (1999) aluden a que existen investigaciones que avalan las posibles aplicaciones de la investigación de la parapsicología en el campo de la medicina, tecnología, política, etc. De este modo, incluso Di Liscia (1990) considera que habría que avanzar en el conocimiento de la influencia de la creación artística tras una experiencia paranormal.

El objetivo principal de este estudio fue determinar la relación existente entre la creatividad, el grado de deterioro cognitivo y las creencias paranormales en un grupo de ancianos de un centro institucionalizado. Asimismo, se ha analizado si existían diferencias en las variables anteriormente mencionadas atendiendo a características sociodemográficas (sexo, edad y estado civil).

\section{MÉTODO}

\section{Participantes}

Para este estudio se utilizó un muestreo intencional. Los criterios de inclusión utilizados fueron los siguientes: ser residentes en la institución seleccionada y presentar un nivel máximo de deterioro cognitivo moderadamente grave o demencia moderada (GDS 5) según la Escala de Deterioro Global (GDS- Global Deterioration Scale-, de 
Reisberg, Ferris, León, y Crook, 1982), para facilitar que pudiesen responder a los instrumentos de evaluación. Como criterios de exclusión se tuvieron en cuenta: tener un nivel de deterioro global que denotase demencia moderadamente grave o grave (GDS 6 o 7) lo que imposibilitaría responder los instrumentos utilizados.

Atendiendo a los criterios comentados, participaron en el estudio un grupo de 36 personas mayores, de los 79 del total que residen en el centro institucionalizado seleccionado de Murcia con edades comprendidas entre los 66 y 95 años $(M=80.86$ años; $D T=7.36)$. En lo que respecta al sexo hubo 16 varones. En lo referente al estado civil había: $16.7 \%$ de solteros, $19.4 \%$ de casados, $55.6 \%$ de viudos y $8.3 \%$ de separados/ divorciados.

\section{Instrumentos}

Se administraron tres instrumentos para recabar información sobre el deterioro cognitivo, la creatividad y las creencias paranormales. A continuación se describe brevemente cada uno de ellos:

Para valorar el estado cognitivo se utilizó el Mini-Examen Cognoscitivo (MEC-35) elaborado por Lobo et al. (1979) es la adaptación española del Mini-Mental State Examination (MMSE) de Folstein, Folstein, y McHugh (1975). Es un test de cribado que permite detectar el deterioro cognitivo. Consta de 35 ítems que examinan las siguientes funciones cognitivas: orientación, memoria, atención, cálculo, lenguaje, construcción, praxias y razonamiento. La puntuación final se establece tras llevar a cabo la correción dependiendo del número de ítems que hayan sido eliminados por analfabetismo o por imposibilidad física de cumplimentar el ítem (por ejemplo, en caso de ceguera). El punto de corte para personas mayores de 65 años en un geriátrico se sitúa en 23 puntos.

Para evaluar la creatividad se utilizó el Test CREA (Inteligencia Creativa. Una medida Cognitiva de la Creatividad) elaborado por Corbalán Berná et al. (2003). El instrumento ofrece una apreciación de la inteligencia creativa a través de una evaluación de tipo cognitiva de la creatividad individual. El CREA proporciona una medida única que correlaciona con los factores que influyen en la creatividad (Fluidez, Flexibilidad, Originalidad y Producción Divergente). El test consta de tres láminas estímulo (A, B y C) a partir de las cuales los sujetos han de formular la mayor cantidad de interrogantes durante un tiempo delimitado. La Lámina A simboliza un teléfono antiguo y la Lámina B representa una escena que incluye un grupo de personas entre las que una de ella les corta las orejas al resto. Ambas láminas son las que se utilizan en adultos y por lo tanto las seleccionadas para este estudio. La corrección supone la suma de las preguntas realizadas tras la fórmula en la cual se corrige la puntuación obtenida. Para el estudio se ha utilizado la puntuación directa, la puntuación transformada al centil atendiendo a los 
baremos y los percentiles para interpretarlos. Por lo tanto, el test ofrece posibilidades avaladas de medir la creatividad (Corbalán Berná et al., 2003).

Para analizar las creencias paranormales se seleccionó la Escala de Creencias paranormales (Tobacyk, 2004). Consta de 26 ítems que oscilan en los siguientes rangos: 0 mиy en desacuerdo; 1 En desacuerdo; 2 De acuerdo; 3 Muy de acuerdo. El instrumento está formado por 7 dimensiones: creencias religiosas tradicionales, creencias en la parapsicología, brujería, superstición, espiritualismo, formas de vida extraordinarias y precognición. Para la versión revisada se mejoró la confiabilidad temporal y la consistencia interna (Tobacyk, 2004). El índice de fiabilidad alfa de Cronbach para el presente estudio fue de .71.

\section{Procedimiento}

Para llevar a cabo el estudio fue preciso contactar con el Directivo de la institución de personas mayores de Murcia para fijar una reunión durante la cual se explicaría el objetivo del estudio y los instrumentos de evaluación promoviendo su colaboración. Tras la obtención, la selección de los sujetos fue a conveniencia atendiendo a los criterios de inclusión mencionados debido a que era necesario que no tuviesen un nivel de deterioro global compatible con demencia moderadamente grave o grave según la Global Deterioration Scale -Escala de deterioro global- de Reisberg et al. (1982) y que imposibilitase responder a los instrumentos de evaluación. Se aseguró la confidencialidad y el anonimato de los datos recogidos durante todo el proceso. La recogida de datos fue llevada a cabo por los autores del estudio desde agosto del año 2014 hasta enero del año 2015.

\section{Análisis de datos}

Para esta parte de la investigación, el tratamiento estadístico de los datos, se utilizó el programa estadístico informático SPSS, en su versión 19.

Para al análisis de los datos descriptivos, se utilizó el estudio de frecuencias, porcentajes así como medias y desviaciones típicas. En el caso de tratarse de dos variables continuas, utilizamos la correlación de Pearson; si tenía dos niveles en la variable de agrupamiento con respecto a la variable numérica, la comparación de medias entre dos grupos independientes se llevó a cabo a través de la prueba $t$ de Student, pero si tenía tres o más categorías, la comparación de medias entre tres o más factores se realizó a través del análisis de la varianza de un factor (ANOVA). En ambos casos se comprobó el supuesto de homogeneidad de varianzas de Levene. Se utilizó la diferencia de medias tipificada $(d)$ según los valores de Cohen. 


\section{RESULTADOS}

\section{Creatividad y deterioro cognitivo}

En primer lugar en cuanto al nivel cognitivo, cabe destacar que el $38.9 \%$ $(N=14)$ de los ancianos presentaron deterioro cognitivo según el test MEC.

Si analizamos los percentiles del test CREA obtenemos que en la lámina A un sujeto tuvo una puntuación media en creatividad frente al resto que presentaban puntuaciones bajas. Sin embargo, en la lámina B el $8.3 \%$ de sujetos $(N=3)$ tenían niveles medios de creatividad.

$\mathrm{Si}$ atendemos al nivel cognitivo de los sujetos y la creatividad (puntuación directa del test), los resultados mostraron una correlación positiva con efecto medio $(r=.438 ; p=.008 ; N=36)$. Estos resultados son indicativos que a mejor estado cognitivo, mejor nivel de creatividad por lo tanto, puntuaciones bajas en la escala de deterioro cognitivo están indicando una menor creatividad (véase el gráfico 1).

Gráfico 1. Gráfico de dispersión de puntos de la variable creatividad (CREA) y nivel de deterioro cognitivo (MEC-35)

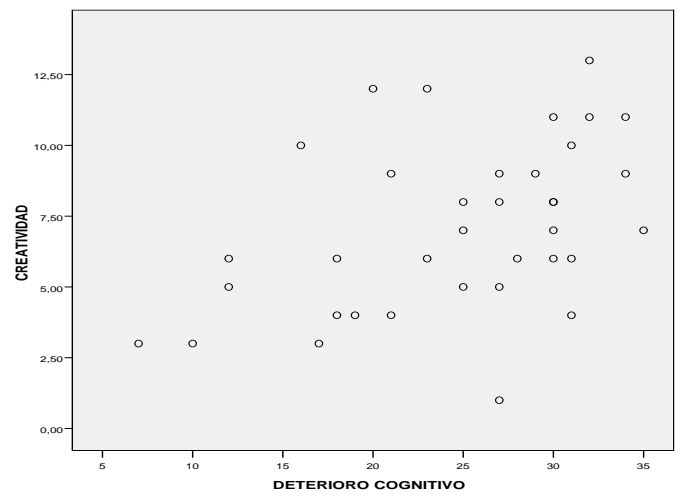

\section{Creatividad y creencias paranormales}

En lo referente a las creencias paranormales y la creatividad, en primer lugar, los resultados determinaron una correlación significativa negativa media entre Creatividad (CREA lámina A) y las Creencias religiosas tradicionales de las personas ( $r=-.393 ; p=.018 ; N=36)$ tal y como puede apreciarse en el gráfico 2.

En segundo lugar, los resultados mostraron una correlación significativa negativa media entre Creatividad (CREA lámina B) y la Superstición ( $r=-.386 ; p=.020$; $N=36$ ) tal y como puede visualizarse en el gráfico 3. 
Gráfico 2. Gráfico de dispersión de puntos de la variable creatividad (CREA A) y Creencias religiosas tradicionales

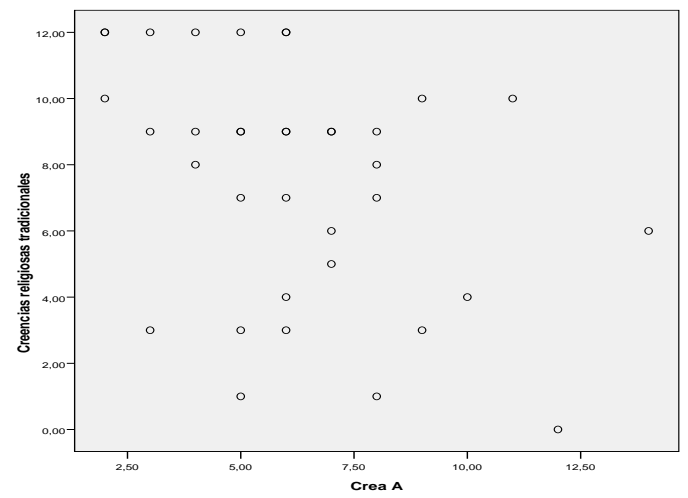

Gráfico 3. Gráfico de dispersión de puntos de la variable creatividad (CREA B) y Superstición

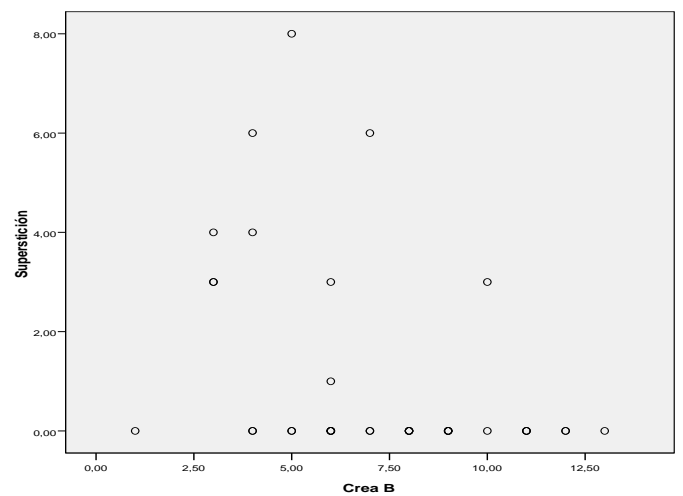

Dado que el $8.3 \%$ de sujetos $(N=3)$ tenían niveles medios de creatividad en la lámina $\mathrm{B}$, según los percentiles, se ha considerado oportuno analizar las diferencias de medias entre los niveles medio y bajo en la prueba t de Student. De este modo, la prueba $t$ de Student mostró diferencias de medias significativas, asumiendo varianzas heterogéneas, en las Creencias en la parapsicología $t_{(11.708)}=3.640 ; p=.004$ con un tamaño del efecto alto $(d=1.22)$. Los sujetos con niveles medios en Creatividad mostraban valores más altos en las Creencias en la parapsicología $(M=4.4 ; D T=4.21)$ que los sujetos con valores más bajos en creatividad $(M=1 ; D T=1)$.

Asimismo, la prueba t de Student mostró diferencias de medias significativas, asumiendo varianzas heterogéneas, en la Superstición $t_{(32.000)}=3.253 ; p=.003$ con un tamaño del efecto alto $(d=1.09)$. Los sujetos con niveles bajos en Creatividad mostraban 
valores más altos $(M=1.24 ; D T=.2 .19)$ en superstición frente a los que obtuvieron niveles medios en Creatividad $(M=.0 ; D T=.0)$.

De otro lado, la prueba $t$ de Student mostró diferencias de medias significativas, asumiendo varianzas heterogéneas, en la Espiritualidad $t_{(32.000)}=5.824$; $p=.000$ con un tamaño del efecto alto $(d=1.95)$. Los sujetos con niveles bajos en creatividad mostraban valores más altos en Espiritualidad $(M=3.72 ; D T=3.68)$ frente a los sujetos con valores medios en creatividad $(M=.0 ; D T=.0)$.

\section{Deterioro cognitivo y creencias paranormales}

Los resultados demostraron una correlación significativa negativa alta entre Deterioro cognitivo (MEC) y la Superstición ( $r=-.603 ; p=.000 ; N=36)$ tal y como puede observarse en el gráfico 4.

Gráfico 4. Gráfico de dispersión de puntos de la Deterioro Cognitivo (MEC) y Superstición

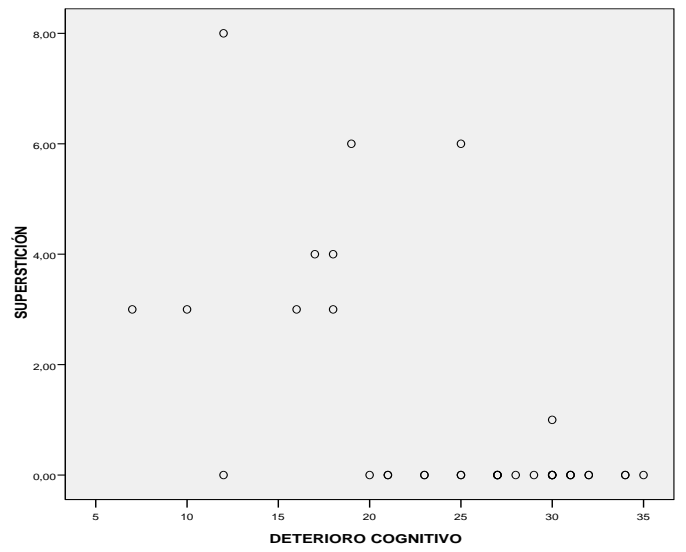

Creatividad, deterioro cognitivo, creencias paranormales según características sociodemográficas

Atendiendo al sexo, la prueba $t$ de Student mostró diferencias de medias significativas, asumiendo varianzas homogéneas, en función del sexo en la Creatividad (lámina A) $t_{(34)}=2.369 ; p=.024$ con un tamaño del efecto medio-alto $(d=.79)$. Los varones mostraron valores más altos en creatividad que las mujeres (véase tabla 1).

En lo que respecta al nivel de deterioro cognitivo (MEC) la prueba $t$ de Student mostró diferencias de medias significativas, asumiendo varianzas homogéneas, en función del sexo $t_{(34)}=-2.624 ; p=.013$ con un tamaño del efecto alto $(d=-.88)$. Los varones obtuvieron un mejor nivel cognitivo que las mujeres tal y como puede apreciarse en la tabla 1. 
Asimismo, la prueba $t$ de Student mostró diferencias de medias significativas, asumiendo varianzas homogéneas, en función del sexo en creencias religiosas tradicionales $t_{(34)}=-4.553 ; p=.000$ con un tamaño del efecto alto $(d=-1.53)$. Las mujeres destacaban en creencias religiosas tradicionales frente a los varones (véase tabla 1). Finalmente, el resto de variables no resultaron ser significativas en lo que respecta al sexo según la prueba $t$ de Student.

Tabla 1. Medias $(M)$ y desviaciones típicas $(D T)$ para los Creatividad, deterioro cognitivo y creencias paranormales en función del sexo

\begin{tabular}{lccc}
\hline & Sexo & $M$ & $D T$ \\
\hline \multirow{2}{*}{ Crea A } & Hombre & 7.38 & 3.09 \\
\cline { 2 - 4 } Crea B & Mujer & 5.30 & 2.15 \\
\hline \multirow{2}{*}{ MEC 35 } & Hombre & 7.50 & 3.43 \\
\cline { 2 - 4 } & Mujer & 6.80 & 2.61 \\
\hline \multirow{2}{*}{$\begin{array}{l}\text { Creencias religiosas } \\
\text { tradicionales }\end{array}$} & Hombre & 27.94 & 5.52 \\
\cline { 2 - 4 } & Mujer & 22.00 & 7.57 \\
\hline \multirow{2}{*}{$\begin{array}{c}\text { Creencia en la } \\
\text { parapsicología }\end{array}$} & Mujer & 9.45 & 2.46 \\
\hline \multirow{2}{*}{ Brujería } & Hombre & 4.50 & 4.38 \\
\cline { 2 - 4 } & Mujer & 3.80 & 4.02 \\
\hline \multirow{2}{*}{ Superstición } & Hombre & 4.00 & 3.16 \\
\hline \multirow{2}{*}{ Espiritualidad } & Mujer & 3.50 & 3.61 \\
\hline \multirow{2}{*}{$\begin{array}{l}\text { Formas de vida } \\
\text { extraordinarias }\end{array}$} & Hombre & 1.00 & 2.19 \\
\hline \multirow{2}{*}{\begin{tabular}{l} 
Precognición \\
\cline { 2 - 4 }
\end{tabular}} & Mujer & 1.25 & 2.12 \\
\hline & Hombre & 2.81 & 3.92 \\
\hline & Mujer & 3.90 & 3.48 \\
\hline
\end{tabular}

Conviene mencionar con respecto a la edad, la existencia de una correlación significativa negativa media con Deterioro cognitivo (MEC) $(r=-.354 ; p=.034 ; N=36)$ no siendo significativa en el resto de variables del estudio.

Por último, cabe mencionar que la variable estado civil no resultó ser significativa en la prueba Anova de un factor para las variables creatividad, deterioro cognitivo y creencias paranormales.

\section{DISCUSIÓN}

Cabe mencionar que 14 personas mayores institucionalizadas del estudio presentaban deterioro cognitivo según el test MEC. Asimismo, únicamente tres sujetos 
presentaron niveles medios de creatividad en la lámina B lo que indicaría que dichos sujetos se caracterizan por presentar un moderado nivel en su producción creativa, no destacando por su capacidad para la innovación o la búsqueda de posibles soluciones a los problemas; mantienen una actitud ante la vida caracterizada por cuestionar parcialmente las situaciones dadas, pudiendo tomar contacto con su potencial para desarrollar sus habillidades creativas. Salvo esto casos, el resto de ancianos se caracterizan por presentar un nivel bajo lo que supone una limitada capacidad para la producción creativa; escasas posibilidades para la búsqueda y el planteamiento de problemas y soluciones alternativas o imaginativas, presentan tendencia general a la adaptación y dificultades para la reflexión crítica (Corbalán et al., 2003).

El análisis del nivel cognitivo de los sujetos y la creatividad indicaron una correlación positiva lo que supone que aquellos sujetos con mejor estado cognitivo son los más creativos. Estos resultados están en la línea con investigaciones que sustentan que la creatividad permite mantener la mente activa así como la imaginación y por tanto previene el deterioro cognitivo en los ancianos (Carrascal y Solera, 2014) mientras que aquellos sujetos que presenten peor estado cognitivo por alguna enfermedad neurodegenerativa, la creatividad se verá afectada (López-Pousa, 2012).

De otro lado, los resultados determinaron una correlación negativa entre Creatividad y las Creencias religiosas tradicionales. Esto supone que aquellas personas con menor creatividad poseían más Creencias religiosas tradicionales. Asimismo, los resultados mostraron una correlación negativa entre Creatividad y la Superstición. Por lo que, a menor creatividad mayor superstición. Asimismo, al analizar las diferencias de medias entre los niveles medio y bajo de creatividad en la prueba t de Student se halló que: los ancianos más creativos mostraban valores más altos en las Creencias en la parapsicología; los ancianos menos creativos mostraban valores más altos en superstición y los ancianos menos creativos obtuvieron valores más altos en Espiritualidad. Estos datos permiten determinar la influencia de la creación artística tras una experiencia paranormal (Di Liscia, 1990).

Los resultados determinaron una correlación negativa entre Deterioro cognitivo y la Superstición, es decir, los ancianos con mejor estado cognitivo eran los menos supersticiosos.

Podemos deducir que la gran mayoría de los ancianos del estudio tuvieron niveles bajos de creatividad y únicamente 14 sujetos presentaron deterioro cognitivo por lo que quizás han buscado apoyo en las creencias religiosas tal y como avalan otros estudios (Reyes-Ortiz, 1998) y se han interesado en temas paranormales debido a experiencias vividas lo que les proporciona interpretaciones más plancenteras de la realidad (Parra y Corbetta, 2013).

Cabe mencionar en cuanto al análisis de las características sociodemográficas, en primer lugar, la prueba $t$ de Student demostró que los varones eran más creativos que 
las mujeres, los varones obtuvieron un mejor nivel cognitivo mientras que las mujeres destacaban en creencias religiosas tradicionales. Es destacable la existencia de una correlación negativa entre Deterioro cognitivo y edad lo que supone que a mayor edad aumenta el nivel de deterioro cognitivo en los ancianos. Finalmente, el estado civil no tuvo influencia sobre la creatividad, el deterioro cognitivo o las creencias paranormales. Estos datos están en la línea con investigaciones previas que avalan la importancia de programas de estimulación cognitiva o de psicoestimulación para mantener las funciones cognitivas evitando el deterioro y fomentado la independencia y la calidad de vida (Carrascal y Solera, 2014; García-Sevilla et al., 2014)

Como conclusiones podríamos mencionar que el estudio ha permitido analizar las relaciones existentes entre la Creatividad de los ancianos de un centro institucionalizado y su Deterioro cognitivo; evaluar las Creencias paranormales de los ancianos y su Creatividad; estimar la relación entre el Deterioro cognitivo y las Creencias paranormales teniendo en cuenta las características sociodemográficas (sexo, edad y estado civil).

El estudio permite reflexionar sobre la importancia de que los profesionales que trabajen con personas mayores lleven a cabo programas que favorezcan la creatividad entre los ancianos a través de actividades artísticas y creativas (por ejemplo, como objetos decorativos de arcilla, complementos o accesorios realizados con telas, etc.) que, partiendo de la observación e interpretación libre de objetos, sea una herramienta que permita mejorar los cambios evolutivos en los niveles cognitivos, sociales y afectivos durante la vejez. En ese sentido, al mismo tiempo que se mantienen las capacidades cognitivas, se pueden programar visitas a museos, exposiciones, recitales, etc. que permitan intercambiar opiniones y por ende favorecer su interacción con el medio (Carrascal y Solera, 2014).

Del mismo modo, sería de utilidad recabar información sobre el sistema de creencias religiosas (Reyes-Ortiz, 1998) así como las creencias paranormales, ya que actúan como amortiguadores de la sintomatología ansiosa o depresiva a la vez que dotan de esperanza y sentido de trascendencia a la persona (Parra y Corbetta, 2013).

Entre las limitaciones del estudio cabe mencionar que se llevó a cabo de forma puntual con un número limitado de participantes. Asimismo, los instrumentos de evaluación fueron dirigidos únicamente a los ancianos, sin recabar información de los profesionales ni de familiares, conocidos o amigos. Nuestro estudio se centró en analizar algunas variables sobre las creencias paranormales, el nivel cognitivo y la creatividad; por lo que, como prospectivas se plantea aumentar el tamaño muestral e incluso añadir variables que proporcionen información sobre: las características de la personalidad, la sintomatología depresiva, ansiedad, la inteligencia emocional (Pérez-Fuentes, Gázquez, Mercader, y Molero, 2014) e incluso la calidad de vida de la persona mayor institucionalizada (Molero, Pérez-Fuentes, Gázquez, y Mercader, 2012). 


\section{REFERENCIAS}

Abellán, M.A. García-Sevilla, J., y Méndez, I. (2013). Diseño de un panel de psicoestimulación como herramienta de intervención en personas mayores con deterioro cognitivo. En J.J. Gázquez, Ma .C. Pérez-Fuentes, M.M. Molero e I. Mercader (Comps.). Calidad de vida, Cuidadores e intervención para la mejora de la salud en el envejecimiento ( $\mathrm{pp} .263-$ 266). Almería: Asociación Universitaria de Educación y Psicología (ASUNIVEP).

Carrascal, S., y Solera, E. (2014). Creatividad y desarrollo cognitivo en personas mayores. Arte, Individuo y Sociedad. 26(1), 9-19.

Corbalán, J., Martínez, F., Donolo, D., Alonso, C., Tejerina, M., y Limiñana, M. (2003). CREA. Inteligencia Creativa. Una medida Cognitiva de la Creatividad. Madrid: TEA Ediciones.

Di Liscia. J.C. (1990). Creatividad artística y percepción extrasensorial, Revista Argentina de Psicología Paranormal, 4, 213-220.

Fernández-Ballesteros, R., Caprara, M.G., Iñiguez, J., y García, L.F. (2005). Promoción del envejecimiento activo: efectos del programa «Vivir con vitalidad. Revista Española de Geriatría y Gerontología, 40(2), 92-102.

Fernández-Ballesteros, R., Zamarrón, M.D., Tárraga, L., Moya, R., y Iñiguez, J. (2003). Cognitive Plasticity in Healthy, Mild cognitive Impairment (MCI) Subjects and Alzheimers Disease Patients: A Research Project in Spain. European Psycologist, 8(3), 148-159. doi: 10.1027//1016-9040.8.3.148

Folstein, M.F., Folstein, S.E., y McHugh, P.R. (1975). "Mini-Mental State": A practical method for grading the cognitive state of patients for the clinician. Journal of Psychiatric Research, 12, 397-408. doi: 10.1016/0022-3956(75)90026-6

Gardner, H. (2010). Mentes Creativas. Barcelona: Paidos Ibérica.

García-Sevilla, J., Fernández, P.J., Fuentes, L.J., López, J.J., y Moreno, M.J. (2014). Estudio comparativo de dos programas de entrenamiento de la memoria en personas mayores con quejas subjetivas de memoria: un análisis preliminar. Anales de Psicología, 30(1), 337-345. doi: 10.6018/analesps.30.1.158021

Gázquez, J.J., Pérez-Fuentes, M.C., Mercader, I., y Molero, M.M. (2011). Prevalencia de la dependencia funcional en personas mayores. Anales de Psicología, 27(3), 871-876.

Krippner, S. (1999). La parapsicología en el siglo veintiuno. Revista Argentina de Psicología Paranormal, 4, 207-220.

López-Pousa, S. (2012). Creatividad pictórica en la demencia asociada a la enfermedad de Parkinson. Revista Alzheimer, 50, 20-29. doi: 10.5538/1137-1242.2012.50.20

Lobo, A., Ezquerra, J., Gómez, F., Sala, J.M., y Seva, A. (1979). El mini-examen cognoscitivo. Un test sencillo y práctico para detectar alteraciones intelectuales en pacientes médicos. Actas Luso Españolas de Neurología y Psiquiatría, 7, 189-201

Molero, M.M., Pérez-Fuentes, M.C., Gázquez, J.J., y Mercader, I. (2012). Construcción y validación inicial de un cuestionario para evaluar la Calidad de Vida en mayores institucionalizados. European Journal of Investigation in Health, Psychology and Education, 2(2), 53-65. doi: 10.1989/ejihpe.v2i2.14

Parra, A., y Corbetta, J.M. (2013). Experiencias paranormales y su relación con el sentido de la vida. Liberabit, 19(2), 251-258.

Pérez-Fuentes, M.C., Molero, M.M., y Gázquez, J.J. (2011). Dependencia y valoración de los Servicios Sociales por los mayores. En M.C. Pérez-Fuentes y J.J. Gázquez (Comps.), Envejecimiento y demencia. Un enfoque multidisciplinar (pp.405-414). Granada: GEU Editorial. 
Pérez-Fuentes, M.C., Gázquez, J.J., Mercader, I., y Molero, M.M. (2014). Brief Emotional Intelligence Inventory for Senior Citizens (EQ-i-M20). Psicothema, 26(4), 524-530. doi: $10.7334 /$ psicothema2014.166

Pérez-Fuentes, M.C., Molero, M.M., Gázquez, J.J., y Soler, F.J. (2014). Estimulación de la Inteligencia Emocional en mayores: El programa PECI-PM. European Journal of Investigation in Health, Psychology and Education, 4(3), 329-339. doi: 10.1989/ejihpe.v4i3.80

Reisberg, B., Ferris, S., de León, M., y Crook, T. (1982). The Global Deterioration Scale (GDS) for the assessment of primary degenerative dementia. American Journal of Psychiatry, 139(9), 1136-1139. doi: 10.1176/ajp.139.9.1136

Reyes-Ortiz, C.A. (1998). Importancia de la religión en los ancianos. Colombia Médica, 29(4), 155-157.

Tobacyk, J. (2004). A Revised Paranormal Belief Scale. The International Journal of Transpersonal Studies, 23, 94-98.

Recibido: 7 de junio de 2015 Recepción Modificaciones: 31 de julio de 2015 Aceptado: 3 de agosto de 2015 